\title{
Generation of circularly polarized photons for a linear collider polarized positron source
}

\author{
V.Strakhovenko $^{a}$, X.Artru $^{b}$, R.Chehab ${ }^{b, c}$, and M.Chevallier ${ }^{b}$, \\ ${ }^{a}$ Budker-INP, 11 Ac.Lavrentyeva, 630090, Novosibirsk, Russia \\ ${ }^{b}$ IPN-Lyon, IN2P3/CNRS et Univ. Claude Bernard, 69622 Villeurbanne, France \\ ${ }^{c}$ LAL, IN2P3/CNRS et Univ. de Paris-Sud, BP 34-91898, Orsay cedex, France
}

\begin{abstract}
Various methods of obtaining longitudinally polarized positrons for future linear colliders are reviewed. Special attention is paid to the schemes using circularly polarized high-energy photons for positron production. Most effectively such photons are obtained from electrons passing through a helical undulator or colliding with a circularly polarized laser wave. Spectrum and polarization of radiation emitted during helical motion of electrons are considered in detail. A new simple presentation of known formulas is used to account for the influence of the wave intensity, of the electron-beam angular divergence, of the collimation of radiation, and of the lateral and temporal profiles of the laser bunch on the radiation properties.
\end{abstract}

PACS numbers: 12.20.Ds , 03.65.Sq

\footnotetext{
${ }^{*}$ Corresponding author, e-mail: v.m.strakhovenko@inp.nsk.su
} 


\section{Introduction}

Systematic studies (see, e.g. 1]) have shown that the use of polarized beams in a $e^{+} e^{-}$linear collider will greatly help in the identification of new particles, in search for new physics and in precision measurements of the coupling parameters. The polarization of the electron beam alone is already very useful in this respect. If the positron beam is also polarized, one can benefit from i) an increase of the effective polarization: for instance, a $80 \% e^{-}$polarization and a $60 \% e^{+}$polarization combine into an effective polarization of $94 \%$; ii) a better precision in the effective and individual polarizations; iii) a further reduction of background events: for instance, the number of $W^{-} W^{+}$pairs is reduced by a factor 2 as compared to the case when only the $e^{-}$beam is polarized; iiii) an increased sensitivity to non-standard couplings. In addition to these improvements, the transverse polarization of both $e^{-}$and $e^{+}$would allow one to investigate $\mathrm{CP}$ violating couplings.

The polarized electron sources have been pioneered at SLAC, since the mid-1970s [2], and used systematically for physics experiment since 1992. The SLC electron beam was polarized to around $80 \%$. Such sources are based on a strained semiconductor photocathode, which absorbs circularly polarized laser photons of energy close to the band gap. State-ofthe-art of polarized electron sources shows that they are presently meeting the linear collider requirements.

All the schemes for obtaining longitudinally polarized positrons (except that using $\beta^{+}$ decay of some isotopes) are based on the reaction $\gamma+\gamma \rightarrow e^{+}+e^{-}$, where at least one of two photons should be circularly polarized. When the $\gamma$-conversion occurs in an amorphous target, the role of the second photon in the reaction is played by an unpolarized Coulomb photon providing the momentum exchange between the created particles and an atom. So, the incident photon should be circularly polarized and have the energy, $\omega$, above the threshold value of $2 m$ ( $m$ is the electron mass, a system of units $\hbar=c=1$ is used ). The helicity transfer at high energy was established in [3] for two basic QED-processes: bremsstrahlung from electrons and $e^{+} e^{-}$pair production by photons. For both processes, the helicity transfer is the most effective in the hard part of the spectra. The pair production cross section $\sigma(\omega)$ increases with $\omega$, e.g., $\sigma(100 \mathrm{MeV}) / \sigma(10 \mathrm{MeV}) \simeq 2.44$ for tungsten. From this point of view, the higher is $\omega$ the larger is the positron yield. However, due to a large phase space of produced positrons, they can be successfully accepted by existing matching systems only if their energy does not exceed several tens of MeV. Another argument, which may be used for a proper choice of $\omega$, is to keep off the main nuclear resonances, thereby diminishing a harmful hadronic background.

If polarized electrons are available, an amorphous target can be used according to results of [3] for subsequent emission of circularly polarized photons and their conversion into polarized $e^{+} e^{-}$pairs. Such a possibility was estimated in [4] for electrons of energy $\varepsilon=50 \mathrm{MeV}$ traversing a thin $\left(\sim 0.2 X_{0}\right)$ amorphous target. Selecting positrons with $\varepsilon>25 \mathrm{MeV}$, a yield of $2 \cdot 10^{-3}$ per one initial electron was found. The mean polarization was about 0.6 of that of the initial electron beam.

When $e^{+} e^{-}$pairs are produced in collision of two real photons, the threshold condition (for head-on collision) reads $\omega_{h} \cdot \omega_{s} \geqslant m^{2}$. Evidently, within this scheme one of the photons is a circularly polarized laser photon, which, typically, is rather soft (e.g., $\omega_{s}=0.117 \mathrm{eV}$ for $\mathrm{CO}_{2}$ laser and $\omega_{s}=2.23 \mathrm{eV}$ for $\mathrm{Nd}$ :glass laser). Unpolarized hard photons may be obtained from high-energy electrons radiating in amorphous or crystal targets. The latter option was considered in [5]. However, from the threshold condition, these photons should be really hard: $\omega_{h}>2.23 \mathrm{TeV}$ for $\mathrm{CO}_{2}$ laser and $\omega_{h}>112 \mathrm{GeV}$ for $\mathrm{Nd}$ :glass laser. In turn, the electron 
energy should be appreciably larger than $\omega_{h}$.

The circularly polarized photons can be emitted by unpolarized electrons under helical motion, which can be realized, in particular, in a helical undulator or in a circularly polarized laser wave. The use of a helical undulator for the production of polarized positrons was proposed first in 6]. Such technique requires incident electrons of very high (of hundreds $\mathrm{GeV}$ ) energy to produce a $\gamma$ beam of tens $\mathrm{MeV}$, since the undulator period is relatively large $(\sim 1 \mathrm{~cm})$. This scheme is proposed in [7] for TESLA LC. It is also considered for the NLC project and a dedicated experiment 8 is to be done at SLAC as a "proof of principle". One of the challenges of this method, which requires a very long $(\sim 200 \mathrm{~m})$ undulator, is an accurate alignment.

A design of a polarized positron source for LC using a circularly polarized laser wave is proposed in [9]. Here the challenges are concerning mainly the laser. Some optimization for the total laser power is worked out and particular efforts are put on the optics. Presently the needed number of $\mathrm{CO}_{2}$ lasers is 10 and the incident beam energy of $5.8 \mathrm{GeV}$ is chosen, providing the maximum $\gamma$ energy of $60 \mathrm{MeV}$. One of the advantages of this method is to have electron and positron main systems independent, as the electron drive beam is of some $\mathrm{GeV}$ compared to the $250 \mathrm{GeV}$ beam considered for TESLA.

Polarization of the emitted photons can be measured using Compton scattering of the polarized photons in a magnetized iron [10]. The same technique may be applied to measure the polarization of created positrons after obtaining from them polarized bremsstrahlung photons in an amorphous radiator.

In the present paper we consider the spectrum and polarization of photons emitted from high-energy electrons under helical motion. The description of the phenomenon is independent of the way how such motion is realized. This is due to the fact that the physics of the process is completely determined by the type of trajectory. Particular attention is paid to the practically important cases of a helical undulator and of a laser wave, which are investigated in detail. We derive a new, rather simple but exact, presentation of known formulas and use it to estimate the influence of the wave intensity, of the electron-beam angular divergence, of the collimation of radiation, and of the lateral and temporal profiles of a laser bunch on the radiation properties.

\section{Characteristics of radiation at helical motion of charged particles}

Let us, first, remind one some results of the so-called quasi-classical operator method (QCOM, see e.g., [11), which are important here. Within this method, which accurately takes into account recoil effects, the radiation characteristics are expressed via the classical trajectory (velocity) of a charged particle in a given external field. At appreciably large energies this is true for a wide class of external fields (see the applicability conditions of QCOM in [12]). Thus, if different field configurations provide similar electron trajectories, radiation will be similar as well, being described by the same formulas. As was emphasized in [13], one pair of such equivalent systems is presented just by a helical undulator and a circularly polarized laser wave. Really, in both cases the transverse momentum, $\boldsymbol{p}_{\perp}(t)$ is

$$
\boldsymbol{p}_{\perp}(t)=<\boldsymbol{p}_{\perp}>+p_{\perp}\left[\boldsymbol{e}_{1} \cos \left(\omega_{0} t\right)+\lambda \boldsymbol{e}_{2} \sin \left(\omega_{0} t\right)\right],
$$

where $\left\langle\boldsymbol{p}_{\perp}\right\rangle$ is a mean value of $\boldsymbol{p}_{\perp}(t)$, so that $\langle\ldots\rangle$ means the averaging over a period of motion, and $\lambda= \pm 1$ corresponds to the sense of rotation of $\boldsymbol{p}_{\perp}(t)$. We use the Cartesian 
basis $\left(\boldsymbol{e}_{1}, \boldsymbol{e}_{2}, \boldsymbol{e}_{3}\right)$, where $\boldsymbol{e}_{3}$ is directed along the electron beam momentum, i.e., parallel to the undulator axis. We suppose that a laser wave propagates towards the beam. Then the "trajectory helicity", $\lambda$, in Eq.(10) coincides with that of the magnetic field in an undulator and is opposite to the helicity of the laser wave.

Another argument in favor of the similarity under discussion was also given in [13. This is a similarity of the undulator electromagnetic field (UF) to the field of a laser wave (LW) in the reference system moving along $\boldsymbol{e}_{3}$ with $V=\left\langle v_{3}>\right.$, where an electron is on the average at rest (RS). Really, in the laboratory system (LS), UF providing the trajectory (11) is purely magnetic and reads

$$
\boldsymbol{H}_{L S}=B\left[\boldsymbol{e}_{1} \cos \zeta+\lambda \boldsymbol{e}_{2} \sin \zeta\right] ; \quad \zeta=k z=(\varkappa x) ; \quad z=x^{3},
$$

where 4 -vector $\varkappa=(0,0,0,-k), k=2 \pi / \lambda_{u}$, and $\lambda_{u}$ is the undulator period. In the electron rest system, we obtain for $\mathrm{UF}$

$$
\begin{aligned}
\boldsymbol{H}_{R S}^{u} & =\frac{\varkappa_{0}^{\prime} B}{k V}\left[\boldsymbol{e}_{1} \cos \zeta+\lambda \boldsymbol{e}_{2} \sin \zeta\right], \\
\boldsymbol{E}_{R S}^{u} & =\varkappa_{0}^{\prime} \frac{B}{k}\left[-\lambda \boldsymbol{e}_{1} \sin \zeta+\boldsymbol{e}_{2} \cos \zeta\right],
\end{aligned}
$$

where $\gamma=\left(1-V^{2}\right)^{-1 / 2}$ and $\varkappa^{\prime}=\gamma k(V, 0,0,-1)$, that is $\varkappa_{0}^{\prime}=\gamma k V \approx \gamma k$. At $\gamma \gg 1$ the electromagnetic field (3) corresponds to the wave of quasi-photons $\left(0 \neq \varkappa^{\prime 2}=k^{2} \ll m^{2}\right)$ propagating towards the electron beam and having the helicity $-\lambda$. In turn, LW with the helicity $-\lambda$ is described in LS by the vector potential

$$
\boldsymbol{A}=a\left[\boldsymbol{e}_{1} \cos \chi+\lambda \boldsymbol{e}_{2} \sin \chi\right], \quad \chi=(\varkappa x), \quad \varkappa=\varkappa_{0}(1,0,0,-1) .
$$

Then in RS the LW-field reads

$$
\begin{aligned}
\boldsymbol{H}_{R S}^{w} & =-\lambda a \varkappa_{0}^{\prime}\left[\boldsymbol{e}_{1} \cos \chi+\lambda \boldsymbol{e}_{2} \sin \chi\right], \\
\boldsymbol{E}_{R S}^{w} & =-\lambda a \varkappa_{0}^{\prime}\left[-\lambda \boldsymbol{e}_{1} \sin \chi+\boldsymbol{e}_{2} \cos \chi\right] .
\end{aligned}
$$

Here $\varkappa_{0}^{\prime}=\gamma \varkappa_{0}(1+V) \approx 2 \gamma \varkappa_{0}$ is the laser photon frequency (energy) in RS. The similarity of two fields in RS is clearly seen from Eqs.(3) and (51).

Radiation in LW depends on two parameters. The intensity parameter, $\xi^{2}$, is expressed via a vector potential: $\xi_{w}^{2}=e^{2}<\boldsymbol{A}^{2}>/ m^{2}$ (e is the electron charge). An expression for this parameter in the case of an undulator may be obtained from comparison of equations (5I) and (3) resulting in $\xi_{u}^{2}=(e B / \mathrm{km})^{2}$. (In the literature dedicated to undulators, the notation $K^{2}$ is usually used for this parameter.) Going back to Eq.(11), we conclude that the amplitude $p_{\perp}=\xi m$, while $\omega_{0}=k$ for an undulator and $\omega_{0}=2 \varkappa_{0}$ for LW. In fact, the argument of oscillating functions in Eq.(11) is $(\varkappa x(t))$. In LS, the phase $(\varkappa x(t))$ goes over into $\omega_{0} t$ at assumed conditions: when the electron energy is appreciably large, $\varepsilon / m \gg 1$, and the transverse velocity of the electron is small $v_{\perp} \ll 1$. The parameter $\xi^{2}$ can be expressed also in terms of the particle trajectory

$$
\xi^{2}=\frac{<\boldsymbol{p}_{\perp}^{2}(t)>-<\boldsymbol{p}_{\perp}(t)>^{2}}{m^{2}},
$$

characterizing thereby the deviation of the trajectory from a straight line. On the other hand, $\xi^{2}$ gives a scale of the interaction strength (see, e.g., p.259 in [11]) in the photon emission. 
In fact, we have roughly $\xi^{2} \approx \alpha N_{\text {int }}$, where $\alpha \simeq 1 / 137$ is the fine structure constant and $N_{\text {int }}$ denotes the number of LW photons (or of quasi-photons in the undulator case) within the interaction volume, $V_{\text {int }}$. The latter is approximately $V_{\text {int }} \sim \lambda_{C}^{2} \lambda_{w}$ since the transverse size of the interaction region is about the Compton wave length, $\lambda_{C}=1 / \mathrm{m} \simeq 3.86 \cdot 10^{-11} \mathrm{~cm}$, and the longitudinal size is about the LW length, $\lambda_{w}$. As known, the photon emission rate is roughly proportional to $\xi^{2}$ for $\xi^{2} \lesssim 1$. From this point of view, larger values of $\xi^{2}$ are more attractive. For a helical undulator, where

$$
\xi_{u}^{2}=\left[0.935 \cdot B(T) \cdot \lambda_{u}(\mathrm{~cm})\right]^{2}
$$

values of $\xi^{2} \sim 0.5 \div 1$ seem achievable. In the laser case, extremely high power density $P$ is needed to reach even $\xi^{2} \sim 0.1$ as

$$
\xi_{w}^{2}=3.66 \cdot 10^{-19} \lambda_{w}^{2}(\mu m) \cdot P\left(W / \mathrm{cm}^{2}\right) .
$$

For example, in 9] a peak $P$ of $2.9 \cdot 10^{15} \mathrm{~W} / \mathrm{cm}^{2}$ is assumed at the focal point, which corresponds to the maximal value $\xi_{\max }^{2} \simeq 0.12$. Note that $\xi_{w}^{2}$ diminishes at the periphery of a laser bunch proportional to the density $n_{w}$ of laser photons $\left(\xi_{w}^{2}=2 \alpha \lambda_{w} \lambda_{C}^{2} n_{w}\right)$.

Radiation in LW depends also on the purely kinematic parameter $s=2(\varkappa p) / \mathrm{m}^{2}$, which appears in the description of the conventional Compton scattering. Recollect that the edge of the Compton spectrum is at $u \equiv \omega /(\varepsilon-\omega)=s$ (or at $x \equiv \omega / \varepsilon=s /(1+s)$ ). We have $s_{u} \simeq 0.95 \cdot 10^{-6} \cdot \varepsilon(\mathrm{GeV}) / \lambda_{u}(\mathrm{~cm})$ for a undulator and $s_{w} \simeq 1.53 \cdot 10^{-2} \cdot \varepsilon(\mathrm{GeV}) \varkappa_{0}(\mathrm{eV})$ for LW. We emphasize that $s_{u} \ll 1$ at any reasonable electron energy $\varepsilon$. A magnitude of $s_{w} \sim 1$ can be easily achieved. However, at $s_{w} \sim 1$ emitted photons will be too hard, since, as explained above, photons in the energy range of several tens of $\mathrm{MeV}$ are needed for effective production of positrons. To meet this condition, noticeably different electron energies should be used in undulators and LW, though $s \ll 1$ in both cases. Since at small $s$ soft photons with $\omega / \varepsilon \sim s \ll 1$ are mainly radiated, the recoil effect may be neglected and the classical description of the process becomes valid.

\subsection{Spectral-angular distribution of radiation}

We can present the differential rate (probability per unite length or time) of photon emission from a unpolarized electron in the following general form

$$
\frac{d N_{\gamma}}{d \Gamma d l}=\frac{1}{2}(A+\boldsymbol{B} \boldsymbol{\zeta}) \equiv \frac{A}{2}(1+\boldsymbol{\eta} \boldsymbol{\zeta}), \quad \boldsymbol{\eta}=\frac{\boldsymbol{B}}{A}
$$

where $\boldsymbol{\eta}$ is the Stokes vector of emitted radiation and the auxiliary vector $\boldsymbol{\zeta}(|\boldsymbol{\zeta}|=1)$ describes the analyzing ability of some ideal photon detector. When we consider the spectral-angular distribution, $d \Gamma=d \omega d \boldsymbol{n}_{\perp}$, where $\boldsymbol{n}_{\perp}=\boldsymbol{k}_{\perp} / \omega$. The quantities $A$ and $\boldsymbol{B}$ are obtained from the appropriate expression for the probability derived within QCOM (e.g., from Eqs. (3) and (4) in [14) if a dependence on time of the transverse momentum (velocity) is specified. When such a dependence is given by Eq.(11), we obtain, doing as in [13]

$$
\begin{aligned}
(A, \boldsymbol{B}) & =\frac{\alpha}{\pi} \sum_{n=1}^{\infty}\left(a^{(n)}, \boldsymbol{b}^{(n)}\right) \delta\left(1+\xi^{2}+y^{2}-\frac{n}{\nu}\right) \\
a^{(n)} & =\frac{\xi^{2} \varphi(u)}{4}\left[J_{n-1}^{2}(Z)+J_{n+1}^{2}(Z)-2 J_{n}^{2}(Z)\right]-J_{n}^{2}(Z)
\end{aligned}
$$




$$
\begin{aligned}
& b_{2}^{(n)}=\frac{\lambda \xi^{2} \varphi(u)}{4} \cdot \frac{1+\xi^{2}-y^{2}}{1+\xi^{2}+y^{2}}\left[J_{n-1}^{2}(Z)-J_{n+1}^{2}(Z)\right] \\
& b_{1}^{(n)}=-b_{l i n}^{(n)} \sin 2 \tilde{\phi}, \quad b_{3}^{(n)}=-b_{l i n}^{(n)} \cos 2 \tilde{\phi} \\
& b_{l i n}^{(n)}=J_{n}^{2}(Z)+\xi^{2}\left[J_{n}^{2}(Z)-J_{n-1}(Z) J_{n+1}(Z)\right] .
\end{aligned}
$$

Here

$$
\begin{aligned}
& \nu=u / s, \quad s=\frac{2(\varkappa p)}{m^{2}}, \quad u=\frac{\omega}{\varepsilon-\omega}, \quad \varphi(u)=1+u+\frac{1}{1+u}, \\
& Z=2 \nu \xi y, \quad y^{2}=\left(\gamma \boldsymbol{n}_{e f}\right)^{2}, \quad \gamma=\varepsilon / m, \quad \boldsymbol{n}_{e f}=\boldsymbol{n}_{\perp}-<\boldsymbol{v}_{\perp}>,
\end{aligned}
$$

$J_{n}(Z)$ are the Bessel functions and the angle $\tilde{\phi}$ is the azimuth of the vector $\boldsymbol{n}_{e f}$. Expression (17) follows from Eqs. (3.5)-(3.9) in [13] and coincides with Eq.(5.23) in 11] if the latter is divided by $\omega$ to pass on from the intensity in [11] to the probability considered here. The only generalization here is in retaining $\left\langle\boldsymbol{v}_{\perp}\right\rangle=\left\langle\boldsymbol{p}_{\perp}\right\rangle / \varepsilon$, which was set to zero in [13] and [1]. The appearance of the $\delta$-function in (7) is due to the assumption that the number of periods in a structure, $N_{p e r}=L / \lambda_{u, w}$ ( $L$ is a length of the undulator or LW bunch) is infinitely large. For large but finite $N_{\text {per }}$, we obtain (see e.g., Eq.(1.148) in [11]) a set of narrow (width of $1 / N_{\text {per }}$ ) peaks at the same points $\nu\left(1+\xi^{2}+y^{2}\right)=n$ as in Eq.(17).

When radiation is emitted along the mean electron velocity, we have $\boldsymbol{n}_{\perp}=\left\langle\boldsymbol{v}_{\perp}\right\rangle$ and $y=Z=0$. Then the sum over $n$ in Eq.(7) reduces to the only term with $n=1$. Moreover, only $J_{n-1}^{2}(Z)$ does not vanish and goes to unity. As a result, the linear polarization vanishes for the forward direction, while the circular polarization is maximal as $\eta_{2} \longrightarrow \lambda$. Then the dependence of the probability on the parameter $\xi^{2}$ becomes linear except for the term $\xi^{2}$ in the argument of the $\delta$-function. In this connection, let us point out that the harmonic's number, $n$, is actually the difference between numbers of LW photons absorbed from and re-emitted into the wave. From angular momentum conservation and some additional consideration it can be shown that only $n=1$ is allowed in the forward direction for the circularly polarized LW. To the same time, $\xi^{2}$ in the $\delta$-function reflects a change of the mean longitudinal velocity due to the interaction with a wave as a classical object, namely, $v_{3}^{2}(t) \simeq 1-\gamma^{-2}-\boldsymbol{v}_{\perp}^{2}(t)$ and $<v_{3}^{2}>\simeq 1-\left(1+\xi^{2}\right) / \gamma^{2}$. That is why the characteristic angle of radiation, $\vartheta_{p h}$, becomes $\vartheta_{p h} \sim \sqrt{1+\xi^{2}} / \gamma$. When $y$ increases, the circular polarization diminishes. It vanishes at $y^{2}=1+\xi^{2}$ and changes its sign at further increase of the emission angle (of the quantity $y$ ).

The first harmonic $(n=1)$ dominates also for $\xi^{2} \ll 1$, where we obtain from Eq.(7) using the notation defined in Eqs.(17) and (8)

$$
\begin{aligned}
\left(A^{(C)}, \boldsymbol{B}^{(C)}\right) & =\frac{\alpha \xi^{2}}{\pi}\left(a^{(C)}, \boldsymbol{b}^{(C)}\right) \delta\left(1+y^{2}-\frac{1}{\nu}\right) ; \quad b_{\text {lin }}^{(C)}=\nu(1-\nu), \\
a^{(C)} & =\frac{\varphi(u)}{4}-\nu(1-\nu), \quad b_{2}^{(C)}=\frac{\lambda \varphi(u)}{4} \cdot \frac{1-y^{2}}{1+y^{2}}=\frac{\lambda \varphi(u)}{4}(2 \nu-1) .
\end{aligned}
$$

As should be, Eq.(9) reproduces well known formulas obtained within the Born approximation of the perturbation theory in $\xi^{2}$ for the Compton effect. The latter is usually described in 
terms of a cross section which is obtained from Eq. (9) after dividing by the flux. More precisely, we have at $\xi^{2} \ll 1$

$$
\frac{d \sigma^{(C)}}{d \Gamma}=\frac{4 \pi \alpha \varepsilon}{m^{2} \xi^{2}(\varkappa p)} \cdot \frac{d N_{\gamma}^{(C)}}{d \Gamma d l}
$$

Using the following relation (see 8.442 in [15])

$$
J_{n}^{2}(z)=\sum_{k=n}^{\infty}(-1)^{k-n}\left(\frac{z}{2}\right)^{2 k} \frac{C_{2 k}^{k-n}}{(k !)^{2}} ; \quad C_{N}^{M}=\frac{N !}{M !(N-M) !}
$$

we can expand the quantities $A, \boldsymbol{B}$ defined by Eq.(17) in powers of the parameter $\xi^{2}$

$$
\begin{aligned}
(A, \boldsymbol{B}) & =\frac{\alpha}{\pi} \xi^{2} \sum_{n=1}^{\infty} \delta\left(1+\xi^{2}+y^{2}-\frac{n}{\nu}\right) \sum_{k=n}^{\infty}(-1)^{k-n} \frac{C_{2 k}^{k-n}(\nu \xi y)^{2(k-1)}}{[(k-1) !]^{2}}\left(a^{(k)}, \boldsymbol{b}^{(k)}\right) \\
a^{(k)} & =\frac{\varphi(u)}{4}-\left(\frac{\nu y}{k}\right)^{2}, \quad b_{2}^{(k)}=\frac{\lambda \nu \varphi(u)}{4 k}\left(1+\xi^{2}-y^{2}\right) ; \\
b_{1}^{(k)} & =-b_{\text {lin }}^{(k)} \sin 2 \tilde{\phi}, \quad b_{3}^{(k)}=-b_{\text {lin }}^{(k)} \cos 2 \tilde{\phi}, \quad b_{\text {lin }}^{(k)}=\left(\frac{\nu y}{k}\right)^{2}\left[1+\frac{\xi^{2}}{k+1}\right] .
\end{aligned}
$$

Such presentation of the probability (6) essentially simplifies calculations for $\xi^{2} \lesssim 1$, which is the most interesting region in applications. The formulas obtained above should be averaged over $\left\langle\boldsymbol{v}_{\perp}\right\rangle$ using a corresponding distribution in the incident electron beam.

\subsection{Spectral characteristics of radiation}

In practice, radiation is always collimated. Then, to obtain the spectrum and polarization of photons accepted by a collimator, we should take an integral of the following type

$$
\int_{\Omega_{c}} d \boldsymbol{n}_{\perp} \int d \boldsymbol{\theta}_{e} f\left(\boldsymbol{\theta}_{e}\right)(A, \boldsymbol{B})
$$

where $\Omega_{c}$ is the solid angle of a collimator, $f\left(\boldsymbol{\theta}_{e}\right)$ is the distribution over $\left\langle\boldsymbol{v}_{\perp}\right\rangle$ (we have introduced $\boldsymbol{\theta}_{e} \equiv<\boldsymbol{v}_{\perp}>$ ) normalized by $\int d \boldsymbol{\theta}_{e} f\left(\boldsymbol{\theta}_{e}\right)=1$, and $A, \boldsymbol{B}$ are defined by Eq. (17) or Eq.(11). Below we consider a round collimator with the opening angle $\vartheta_{\text {col }}$ and axially symmetric distributions $f\left(\boldsymbol{\theta}_{e}\right) \equiv f\left(\boldsymbol{\theta}_{e}^{2}\right)$. Remember that the quantities $A, \boldsymbol{B}$ depend on the angles $\boldsymbol{n}_{\perp}$ and $\boldsymbol{\theta}_{e}$ only in the combination (see (8) ) $\boldsymbol{n}_{e f}=\boldsymbol{n}_{\perp}-\boldsymbol{\theta}_{e}$. The azimuth of $\boldsymbol{n}_{e f}$ enters only the linear polarization via $\sin 2 \tilde{\phi}$ and $\cos 2 \tilde{\phi}$, while the value of $\boldsymbol{n}_{e f}^{2}$ is fixed by $\delta$-function $\left(\left(\gamma \boldsymbol{n}_{e f}\right)^{2}=y^{2}=n / \nu-\left(1+\xi^{2}\right)\right)$. After the shift $\boldsymbol{\theta}_{e} \longrightarrow \tilde{\boldsymbol{\theta}}_{e}+\boldsymbol{n}_{\perp}$ in Eq. (12) the integration over $\boldsymbol{\theta}_{e}$ becomes trivial under our assumptions. Really, the linear polarization vanishes at integration over azimuth and integration over $\left|\tilde{\boldsymbol{\theta}}_{e}\right|$ is carried out using $\delta$-function. As a result, we have

$$
\frac{d^{2} N_{\gamma}}{d \omega d l}=\frac{A^{(\omega)}}{2}\left(1+\boldsymbol{\eta}^{(\omega)} \boldsymbol{\zeta}\right), \quad \boldsymbol{\eta}^{(\omega)}=\frac{\boldsymbol{B}^{(\omega)}}{A^{(\omega)}} ; \quad B_{1}^{(\omega)}=B_{3}^{(\omega)}=0
$$




$$
\begin{gathered}
\left(A^{(\omega)}, B_{2}^{(\omega)}\right)=\frac{\alpha \xi^{2}}{\gamma^{2}} \sum_{n=1}^{\infty} \Theta\left(X_{n}\right) F\left(X_{n}, \vartheta_{c o l}\right) \sum_{k=n}^{\infty}(-1)^{k-n} \frac{C_{2 k}^{k-n}\left(\nu \xi^{2} X_{n}\right)^{(k-1)}}{[(k-1) !]^{2}}\left(a^{(\omega)}, b_{2}^{(\omega)}\right) ; \\
a^{(\omega)}=\frac{\varphi(u)}{4}-\frac{\nu X_{n}}{k^{2}}, \quad b_{2}^{(\omega)}=\frac{\lambda \varphi(u)}{4 k}\left[2 \nu\left(1+\xi^{2}\right)-n\right], \quad X_{n}=n-\nu\left(1+\xi^{2}\right) .
\end{gathered}
$$

where $\Theta\left(X_{n}\right)$ is the step function: $\Theta(x)=1$ for $x>0$ and $\Theta(x)=0$ for $x<0$, the variable $\nu \propto \omega$ is defined in (8) . The function $F\left(X_{n}, \vartheta_{c o l}\right)$, which appears in integration over $d \boldsymbol{n}_{\perp}$ in Eq.(12), has the form

$$
F\left(X_{n}, \vartheta_{c o l}\right)=\int d \boldsymbol{n}_{\perp} \Theta\left(1-\frac{\boldsymbol{n}_{\perp}^{2}}{\vartheta_{c o l}^{2}}\right) f\left(\boldsymbol{n}_{\perp}+\boldsymbol{e} \mu \vartheta_{c o l}\right), \quad \mu=\frac{1}{\gamma \vartheta_{c o l}} \sqrt{\frac{X_{n}}{\nu}}
$$

where $\boldsymbol{e}$ is an arbitrary unit vector in the transverse plane. When the angular spread $\Delta_{e}$ in the electron beam is appreciably small, $\Delta_{e} \ll \vartheta_{c o l}$, we can substitute $\left(\mu \vartheta_{c o l}\right)^{2}$ for $\boldsymbol{n}_{\perp}^{2}$ in the argument of $\Theta$-function in (14). Then, using the normalization condition for the function $f$, we find out that $F\left(X_{n}, \vartheta_{c o l}\right) \longrightarrow \Theta\left(1-\mu^{2}\right)$ at $\Delta_{e} \ll \vartheta_{c o l}$. In this case, the contribution of the $n$ - th harmonic to the spectrum is non-zero in the segment

$$
\frac{1}{1+\xi^{2}+\left(\gamma \vartheta_{c o l}\right)^{2}} \leqslant \frac{\nu}{n} \leqslant \frac{1}{1+\xi^{2}}, \quad \frac{\omega}{\varepsilon}=\frac{s \nu}{1+s \nu} .
$$

In the absence of collimation, which corresponds to the limit $\vartheta_{\text {col }} \longrightarrow \infty$ in above formulas, we obtain the evident result, $F\left(X_{n}, \vartheta_{c o l}\right)=1$, being independent of $\Delta_{e}$. Generally speaking, in this case it is more convenient to calculate the spectrum and the rate, $d N_{\gamma} / d l=\int_{0}^{\varepsilon} d \omega A^{(\omega)}$, using simple integral representation for these quantities (see e.g., Eq.(5.34) in [11]). In particular, we have for small $s \ll 1$

$$
\frac{d N_{\gamma}}{d l}(s \ll 1) \simeq \frac{\alpha(\varkappa p) \xi^{2}}{\pi \varepsilon} \int_{0}^{\infty} \frac{d \tau}{\tau^{2}} \cdot \frac{\sin ^{2} \tau-\tau^{2} \cos 2 \tau}{\tau^{2}+\xi^{2}\left(\tau^{2}-\sin ^{2} \tau\right)} .
$$

We emphasize that, for $s \ll 1$, the rate turns out to be independent of the electron energy $\varepsilon$ as $(\varkappa p) / \varepsilon=\omega_{0}$ (remember that $\omega_{0}=2 \varkappa_{0}$ for LW). The first term in the expansion of the rate (16) in $\xi^{2}$ at $\xi^{2} \ll 1$ is simply $W_{0} \equiv 2 \alpha \xi^{2}(\varkappa p) / 3 \varepsilon$ and, using Eq.(10), the Thomson limit of the Compton-scattering cross section $\sigma^{(C)}(s \ll 1) \simeq 8 \pi \alpha^{2} / 3 m^{2} \simeq 665 m b$ is reproduced. The rate (16) is shown in Fig 1 in units of $W_{0}$

The intensity of radiation, $I_{\gamma}$, reads especially simple for $s \ll 1$ when the classical formula is valid

$$
I_{c l}=I_{\gamma}(s \ll 1)=\frac{\alpha \xi^{2} m^{2} s^{2}}{6} \equiv \frac{2}{3} \gamma^{4}<w^{2}(t)>,
$$

where $w(t)$ is the particle acceleration. The radiative energy loss of an electron is determined by $I_{\gamma}$.

In further analysis of the role of photon collimation and angular divergence in the electron beam we will use the Gaussian shape for the function $f$

$$
f_{G}=\frac{1}{2 \pi \Delta_{e}^{2}} \exp \left(-\frac{\boldsymbol{\theta}_{e}^{2}}{2 \Delta_{e}^{2}}\right)
$$




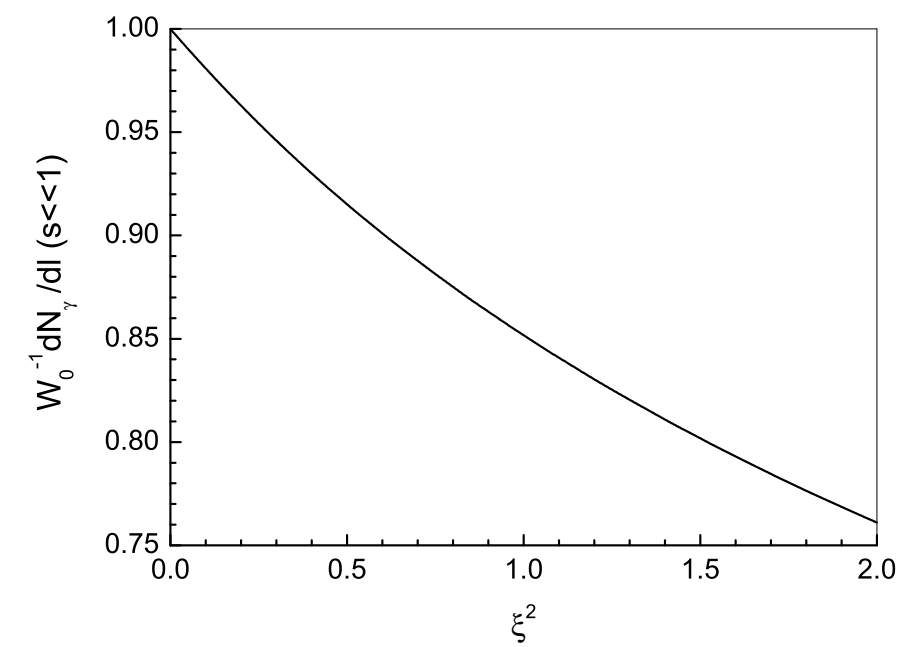

Figure 1: Photon emission rate (16) in units of $W_{0}$.

Then we obtain from Eq.(14)

$$
\begin{aligned}
F_{G}(\beta, \mu) & =2 \beta \int_{0}^{1} d x x \exp \left[-\beta\left(x^{2}+\mu^{2}\right)\right] I_{0}(2 \beta \mu x)= \\
& =\Theta\left(1-\mu^{2}\right)-\frac{1}{\pi} \int_{0}^{\pi} \frac{d \phi}{g(\phi)}(1+\mu \cos \phi) \exp [-\beta g(\phi)] ; \\
g(\phi) & =1+2 \mu \cos \phi+\mu^{2}, \quad \mu^{2}=\frac{X_{n}}{\nu\left(\gamma \vartheta_{c o l}\right)^{2}}, \quad \beta=\frac{\vartheta_{c o l}^{2}}{2 \Delta_{e}^{2}},
\end{aligned}
$$

where $I_{0}$ is the modified Bessel function. The first form of $F_{G}(\beta, \mu)$ in Eq. (17) follows directly from Eq.(14), the second form makes more obvious the general properties of $F\left(X_{n}, \vartheta_{\text {col }}\right)$ discussed above. Shown in Fig. 2 is $F_{G}(\beta, \mu)$ as a function of $\mu$ at different $\beta$. As seen in Fig 2, the function $F_{G}(\beta, \mu)$ is very like to that for a non-divergent beam already at $\beta=25$. At $\beta=4$ the shape of the corresponding curve is substantially different from $\Theta\left(1-\mu^{2}\right)$, but its value at $\mu=0$ (peak position) is still very close to 1 . Finally, at $\beta=1$ we have $F_{G}(\beta=1, \mu=0) \simeq 0.63$ and a slow decrease of $F_{G}$ when $\mu$ increases. In other words, collimation of radiation within angles comparable with the angular divergence of an electron beam is meaningless.

The spectrum summed up over photon polarizations given by $A^{(\omega)}$ in Eq. (13), is plotted in Figs 3 and 4 as a function of $\tilde{\nu}=\nu\left(1+\xi^{2}\right)$. The component $\eta_{2}^{(\omega)}=B_{2}^{(\omega)} / A^{(\omega)}$ of the Stokes vector, which describes the circular polarization of photons is presented as well. The kinematic parameter $s$ (see Eq.(8) ) was set to $s=0.01$, when the normalization factor $W_{\text {noc }}$ is given by Eq.(16). Calculations are performed at $\lambda=-1$ (see Eq.(11) for the definition of $\lambda$ ), which corresponds to the positive helicity of LW and to the negative "helicity" of the electron trajectory in an undulator or LW. Radiation spectra are presented for $\xi^{2}=0.1$ (a) and for $\xi^{2}=1$ (b). In Fig 3, radiation characteristics in the absence of collimation (solid curves) are compared with those at collimation within $\vartheta_{\text {col }}$ satisfying the condition $\left(\gamma \vartheta_{c o l}\right)^{2}=1+\xi^{2}$ for a non-divergent electron beam (dashed curves). At chosen collimation angle, the contribution 


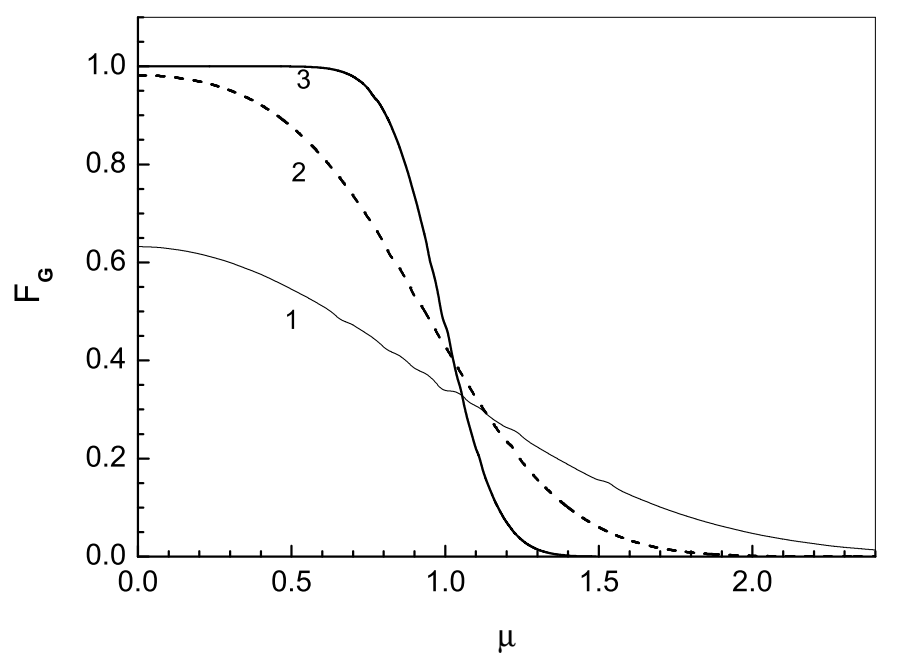

Figure 2: Function $F_{G}(\beta, \mu)$ at $\beta=1(1), \beta=4(2)$, and $\beta=25$ (3) .

of the n-th harmonic (for a non-divergent electron beam) is non-zero for $n / 2 \leqslant \tilde{\nu} \leqslant n$. This leads to corresponding structures in collimated spectra, which are clearly seen in Fig, 3 , Since higher harmonics are more important at $\xi^{2}=1$, reduction of the first peak height and increase of polarization degree are more prominent in this case. A role of the beam divergence is illustrated by Fig 4, where spectra and polarizations are plotted for the same $\vartheta_{c o l}$ as in Fig 3, but at finite values of the parameter $\beta$ (see Eq.(17)). In accordance with Fig 2, structures at $\tilde{\nu}=n / 2$ are smeared out and additional suppression of the first peak at $\beta=1$ is seen even for $\xi^{2}=0.1$.

\subsection{Total yield}

A total radiation yield during one pass of an electron through the whole undulator or a single laser bunch is obtained by integration of the instantaneous characteristics over time (length $l$ ). The electron energy $\varepsilon$ diminishes due to the radiative energy loss given by the radiation intensity $I_{\gamma}$. In Eq.(13),$\varepsilon$ is represented explicitly $\left(\gamma^{-2}\right.$ as a common multiplier and in the quantity $\mu$ entering $\left.F\left(X_{n}, \vartheta_{c o l}\right)\right)$ and implicitly via $\nu$ and $u$. However, the total energy loss and the energy spread in the electron beam is typically small as compared with $\varepsilon$ and therefore will be neglected below. In the opposite case of the appreciably large energy loss, total radiation should be described in terms of the electron-photon shower, which development is determined by the probabilities obtained above. Strictly speaking, the collimation angle $\vartheta_{\text {col }}$ also depends on $l$. Practically this is important only in the case of an undulator when the total length, $L$, is comparable with the distance to a target. In the case of LW we can neglect a variation of $\vartheta_{c o l}$ over $L$ as a laser bunch is usually very short. The parameter $\xi^{2}$ is constant for an undulator and depends on $l$ for LW.

Thus, the total spectral yield from an undulator, $d N_{\gamma} / d \omega$, is given within our approximation by Eq.(13), if we multiply the quantities $A^{(\omega)}, B_{2}^{(\omega)}$ by $L$ and substitute $\Phi=$ $L^{-1} \int_{0}^{L} d l F\left(X_{n}, \vartheta_{c o l}(l)\right)$ for $F\left(X_{n}, \vartheta_{c o l}\right)$. In fact, we should perform the integration of $\Theta$ - 

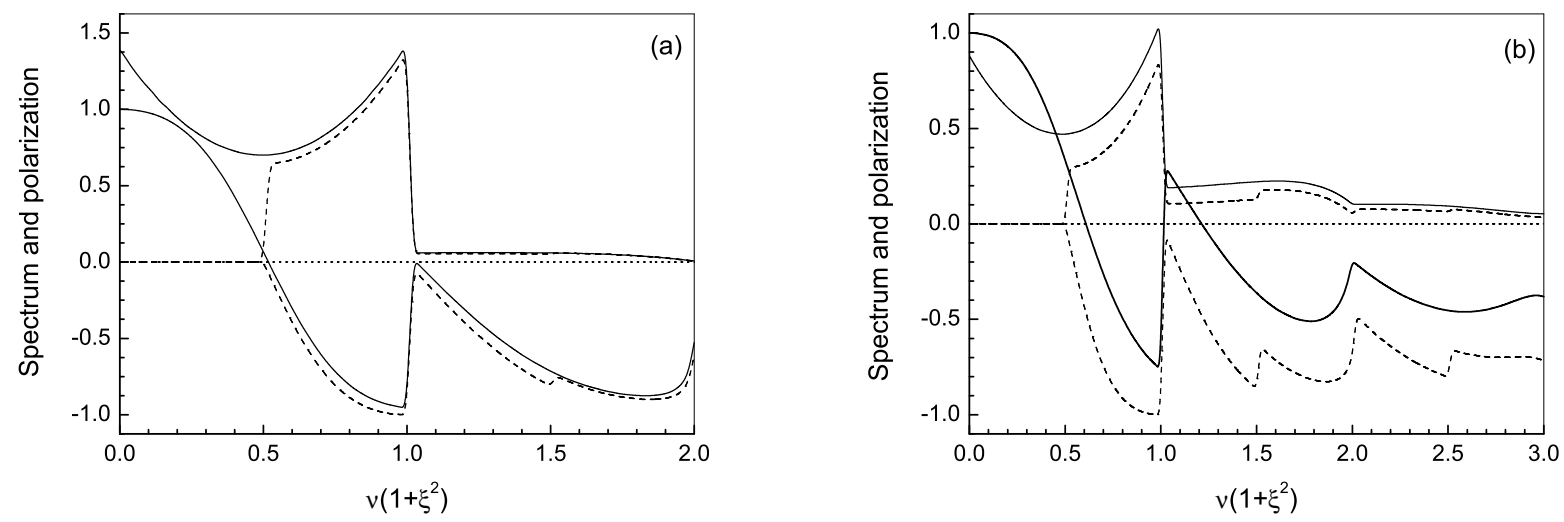

Figure 3: For $\xi^{2}=0.1$ (a) and $\xi^{2}=1(\mathrm{~b})$; spectrum $W_{n o c}^{-1} \cdot d^{2} N_{\gamma} / d \tilde{\nu} d l$ and polarization $\eta_{2}^{(\omega)}$ in the absence of collimation (solid), and at collimation within $\vartheta_{c o l}$ satisfying $\left(\gamma \vartheta_{c o l}\right)^{2}=1+\xi^{2}$ for a non-divergent electron beam (dashed).
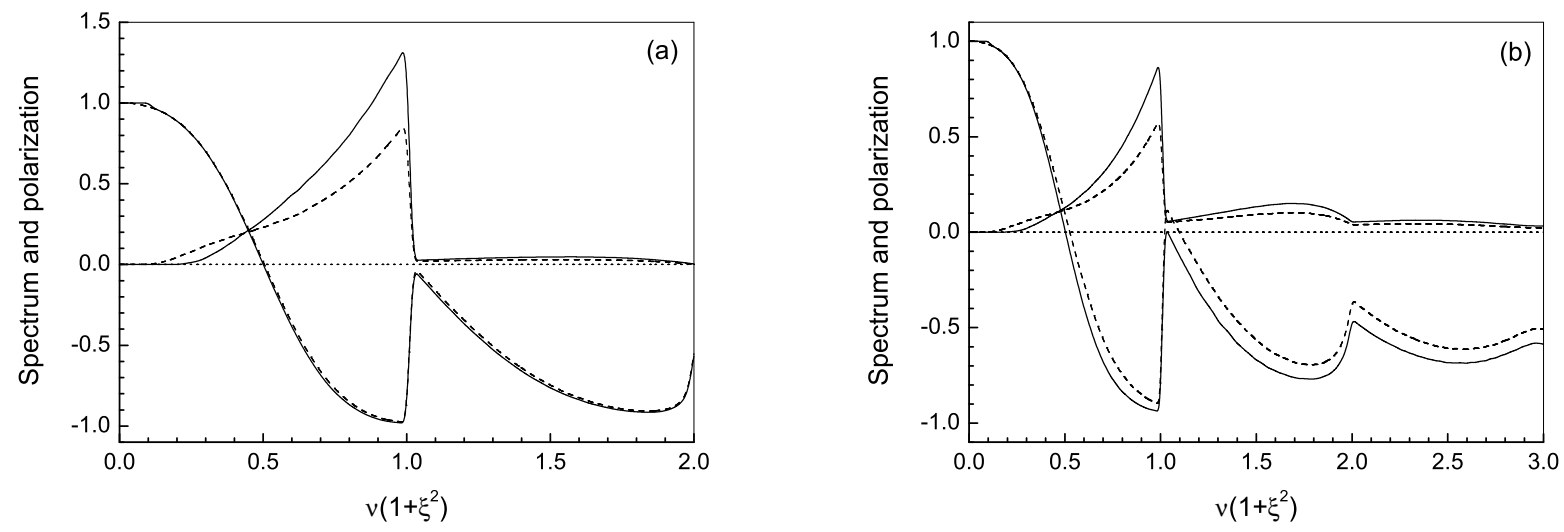

Figure 4: Same as in Fig 3 at the collimation within $\vartheta_{c o l}$ satisfying $\left(\gamma \vartheta_{c o l}\right)^{2}=1+\xi^{2}$ for a divergent electron beam at $\beta=1$ (dashed) and $\beta=4$ (solid).

function in Eq.(14):

$$
\int_{0}^{L} \frac{d l}{L} \Theta\left(1-\frac{\boldsymbol{n}_{\perp}^{2}}{\vartheta_{\text {col }}^{2}(l)}\right)=\Theta\left(1-\frac{\boldsymbol{n}_{\perp}^{2}}{\vartheta_{\text {in }}^{2}}\right)+\Theta\left(1-\frac{\boldsymbol{n}_{\perp}^{2}}{\vartheta_{\text {out }}^{2}}\right) \Theta\left(\frac{\boldsymbol{n}_{\perp}^{2}}{\vartheta_{\text {in }}^{2}}-1\right)\left[\frac{\vartheta_{\text {out }}}{\left|\boldsymbol{n}_{\perp}\right|}-1\right] \frac{\vartheta_{\text {in }}}{\vartheta_{\text {out }}-\vartheta_{\text {in }}}
$$

where $\vartheta_{\text {in }}=\vartheta_{\text {col }}(0)$ and $\vartheta_{\text {out }}=\vartheta_{\text {col }}(L)$ are the angles $\left(\vartheta_{\text {out }}>\vartheta_{\text {in }}\right)$ at which a collimator is seen from the entrance and the exit of the undulator, respectively. Using this result, the integration over $\boldsymbol{n}_{\perp}$ in Eq.(14) can be performed. In particular, we obtain for the Gaussian type of $f$ in Eq.(14)

$$
\begin{aligned}
\Phi_{G} & =2 Q\left\{\int_{0}^{\mu_{\text {in }}^{-1}} d x x \exp \left[-Q\left(x^{2}+1\right)\right] I_{0}(2 Q x)+\int_{\mu_{\text {in }}^{-1}}^{\mu_{\text {out }}^{-1}} d x \frac{1-x \mu_{\text {out }}}{\mu_{\text {in }}-\mu_{\text {out }}} \exp \left[-Q\left(x^{2}+1\right)\right] I_{0}(2 Q x)\right\} \\
\Phi_{\text {nod }} & =\Theta\left(1-\mu_{\text {in }}^{2}\right)+\Theta\left(1-\mu_{\text {out }}^{2}\right) \Theta\left(\mu_{\text {in }}^{2}-1\right) \frac{1-\mu_{\text {out }}}{\mu_{\text {in }}-\mu_{\text {out }}}
\end{aligned}
$$




$$
\mu_{\text {in }}=\frac{1}{\gamma \vartheta_{\text {in }}} \sqrt{\frac{X_{n}}{\nu}}, \quad \mu_{\text {out }}=\frac{1}{\gamma \vartheta_{\text {out }}} \sqrt{\frac{X_{n}}{\nu}}, \quad Q=\frac{X_{n}}{2 \nu\left(\gamma \Delta_{e}\right)^{2}} .
$$

Here $\Phi_{\text {nod }}$ represents the limit of $\Phi_{G}$ at $Q \longrightarrow \infty$ which corresponds to the non-divergent beam. Note also that the first item in $\Phi_{G}$ coincides with $F_{G}\left(\beta_{i n}, \mu_{i n}\right)$ from Eq.(17) and that $Q=\beta_{\text {in }} \mu_{\text {in }}^{2}=\beta_{\text {out }} \mu_{\text {out }}^{2}$ is independent of the collimation angle. In the absence of the external collimator its role is played by the aperture of an undulator. Thus, radiation which escapes the undulator may be described using $\Phi_{G}$ from Eq.(19), where we should set $\vartheta_{\text {in }}=\arctan (r / L) \sim r / L(r$ is the aperture radius $)$ and tend $\mu_{\text {out }}$ to zero.

As explained above, the electron energy and collimation angle may be considered as constant during collision of an electron beam with a laser bunch. Thus, a variation of the parameter $\xi^{2}$ only should be taken into account when obtaining the total yield. Moreover, since $\xi^{2}$ depends also on coordinates $\boldsymbol{r}$ a convolution should be performed with a corresponding distribution $n_{e}(\boldsymbol{r}, t)$ in the electron beam. Let $G\left(\xi^{2}\right)$ is some instantaneous characteristics of radiation, for example, $G\left(\xi^{2}\right)=A^{(\omega)}$ from Eq. (13)), if we consider the spectrum. Then the corresponding total yield, $G_{\text {out }}$, reads

$$
G_{\text {out }}=\int_{-\infty}^{\infty} d t \int d \boldsymbol{r} n_{e}(\boldsymbol{r}, t) G\left(\xi^{2}(\boldsymbol{r}, t)\right) .
$$

This expression greatly simplifies for $\xi^{2} \ll 1$ when (see, e.g, Eq.(9)) the rather complicated, non-linear dependance of $G\left(\xi^{2}\right)$ on $\xi^{2}$ reduces to linear one: $G\left(\xi^{2} \ll 1\right) \simeq \xi^{2} G_{0}$, where $G_{0}$ is independent of $\xi^{2}$. As explained above, we really have $\xi^{2} \ll 1$ for lasers. Then, recollecting the interrelation between $\xi^{2}$ and the laser photon density $n_{w}$, which reads $\xi^{2}=4 \pi \alpha n_{w} /\left(\varkappa_{0} m^{2}\right)$, we can rewrite Eq.(20)

$$
G_{\text {out }}^{(C)}=\frac{4 \pi \alpha G_{0}}{\varkappa_{0} m^{2}} \int_{-\infty}^{\infty} d t \int d \boldsymbol{r} n_{e}(\boldsymbol{r}, t) n_{w}(\boldsymbol{r}, t) .
$$

For Gaussian shape of the lateral profiles in both electron and laser bunches we have

$$
\begin{aligned}
& n_{e}(\boldsymbol{r}, t)=N_{e} g_{e}\left(z-z_{c}^{(e)}(t)\right) \frac{\exp \left(-\boldsymbol{\rho}^{2} / 2 \sigma_{e}^{2}\right)}{2 \pi \sigma_{e}^{2}}, \quad z_{c}^{(e)}(t)=z_{0}^{(e)}+t, \quad z_{c}^{(w)}(t)=z_{0}^{(w)}-t, \\
& n_{w}(\boldsymbol{r}, t)=N_{w} g_{w}\left(z-z_{c}^{(w)}(t)\right) \frac{\exp \left(-\boldsymbol{\rho}^{2} / 2 \sigma_{w}^{2}(z)\right)}{2 \pi \sigma_{w}^{2}(z)}, \quad \int_{-\infty}^{\infty} d z g_{e, w}(z)=1,
\end{aligned}
$$

where $N_{e}$ and $N_{w}=E_{b} / \varkappa_{0}$ ( $E_{b}$ is the laser-bunch energy) are the total numbers of electrons and photons in corresponding bunches. Functions $g_{e, w}(z)$ characterize the longitudinal (temporal) profiles of the bunches and $z_{c}^{(e, w)}(t)$ mark positions of the bunch centers. Focusing of light, if any, is taken into account by the $z$-dependent width $\sigma_{w}(z)$. The focal point corresponds to $z=0$. Evidently, the yield is maximal when the bunch centers meet just at the focal point, i.e, when $z_{0}^{(w)}+z_{0}^{(e)}=0$. Using densities (22) and making the shift $t \longrightarrow t-z+z_{0}^{(w)}$, we obtain from Eq.(21) for the yield per one collision

$$
G_{\text {out }}^{(C)}=\frac{2 \alpha N_{e} E_{b} G_{0}}{\left(\varkappa_{0} m\right)^{2}} \int_{-\infty}^{\infty} d t g_{w}(t) \int_{-\infty}^{\infty} d z \frac{g_{e}(2 z-t)}{\sigma_{e}^{2}+\sigma_{w}^{2}(z)} .
$$


Remember that for undulators we have presented the yield per one electron. Let $L$ be the total length of the laser pulse. At focusing, the width $\sigma_{w}(z)$ increases rather fast with growing $|z|$, determining a $z$-interval, which contributes to the integral in Eq.(23). When focusing is hard, the size of this interval, $L_{e f}$, becomes noticeably smaller than $L$. Concerning the electronbunch length, the shorter it is, the higher the yield. Anyway, this length should be chosen less or about $L_{e f}$. Diminishing of $\sigma_{e}$ in Eq.(23) increases the yield, the other parameters being constant. However, we should bear in mind that $G_{0}$ depends on $\beta=\vartheta_{\text {col }}^{2} /\left(2 \Delta_{e}^{2}\right)$ (see Eq.(17)) and that the product $\Delta_{e} \cdot \sigma_{e}$ has a lower bound given by the beam emittance. As shown above, $\beta$ should be appreciably large (small $\Delta_{e}$ are needed) to achieve the maximal value of $G_{0}$ at given collimation conditions. Thus, both $\Delta_{e}$ and $\sigma_{e}$ should be small enough, i.e, a low-emittance electron beam is needed.

To perform further calculations in Eq.(23), we should specify a shape of the functions $g_{w}(t)$ and $\sigma_{w}^{2}(z)$. Focusing will be taken into account by choosing

$$
\sigma_{w}^{2}(z)=\sigma_{w}^{2}(0)\left[1+C_{f} y^{2}\right], y=4 z / L .
$$

Let us use for the illustration the temporal profile of a laser bunch considered in [9], where

$$
g_{w}(t)=\frac{4}{3 L}[\Theta(\tau+2) \Theta(-\tau-1)(\tau+2)+\Theta(\tau+1) \Theta(1-\tau)+\Theta(2-\tau) \Theta(\tau-1)(2-\tau)]
$$

with $\tau=4 t / L$. Then, making the shift $z \longrightarrow z+t / 2$, we can take the integral over $t$ in Eq. (23)

$$
\begin{aligned}
G_{\text {out }}^{(C)} & =\frac{8 \alpha N_{e} E_{b} G_{0} R}{3\left(\varkappa_{0} m\right)^{2}\left[\sigma_{e}^{2}+\sigma_{w}^{2}(0)\right]}, \quad R=a^{2} \int_{-\infty}^{\infty} d z g_{e}(2 z) \Psi(z), \quad \Psi(z)=\frac{1}{2} \ln \left[\frac{\left(1+d_{3}^{2}\right)\left(1+d_{4}^{2}\right)}{\left(1+d_{1}^{2}\right)\left(1+d_{2}^{2}\right)}\right]+ \\
& +d_{1} \arctan \left(d_{1}\right)+d_{2} \arctan \left(d_{2}\right)-d_{3} \arctan \left(d_{3}\right)-d_{4} \arctan \left(d_{4}\right) ; \quad d_{1}=(1+y) / a \\
d_{2} & =(1-y) / a, \quad d_{3}=\left(\frac{1}{2}+y\right) / a, \quad d_{4}=\left(\frac{1}{2}-y\right) / a ; \quad a^{2}=\frac{\sigma_{e}^{2}+\sigma_{w}^{2}(0)}{C_{f} \sigma_{w}^{2}(0)}, \quad y=4 \frac{z}{L} .
\end{aligned}
$$

The quantity $R$, which was calculated using $g_{e}(z)=\exp \left(-z^{2} / 2 \sigma_{z}^{2}\right) / \sqrt{2 \pi \sigma_{z}^{2}}$, is shown in Fig as a function of $a^{2}$ at $r=L / \sigma_{z}=3$ (this value of $r$ was used in [9]). At given $a^{2}$ the quantity $R$ increases with $r$. We found out that the lateral profile of a laser beam in [9], which actually is doughnut-shaped (see Fig.10 in [9]), can be satisfactorily approximated in the region around the focal point by the Gaussian one with $\sigma_{w}(0)=19 \mu m$ and $C_{f} \simeq 11$ (see Eq.(24)). Using also $\sigma_{e}=20 \mu m$, we obtain $a^{2}=0.192$ and, correspondingly, $R=0.172$. Now any spectral characteristic of radiation may be estimated for the conditions of [9] using Eq.(25). For example, to estimate the total number of photons emitted during a single collision, $G_{0}=2 \alpha(\varkappa p) / 3 \varepsilon$ ) should be substituted into Eq.(25). In [9], the electron beam subsequently collides with laser bunches at 200 collision points to produce a sufficient number of photons. The whole length of the structure is about $60 \mathrm{~m}$ and there are numerous mirrors and collimators inside. The yield from the whole structure on the $e^{+} e^{-}$-production target is a sum of the individual yields, which are described by Eq.(20) (or by its simplified version (25)). Note that the contribution from each collision point should be calculated at its own collimation angle. The latter is determined by a distance between the collision point and the last (the nearest to the target) collimator. Shown in Fig 6 are spectra (normalized per one 


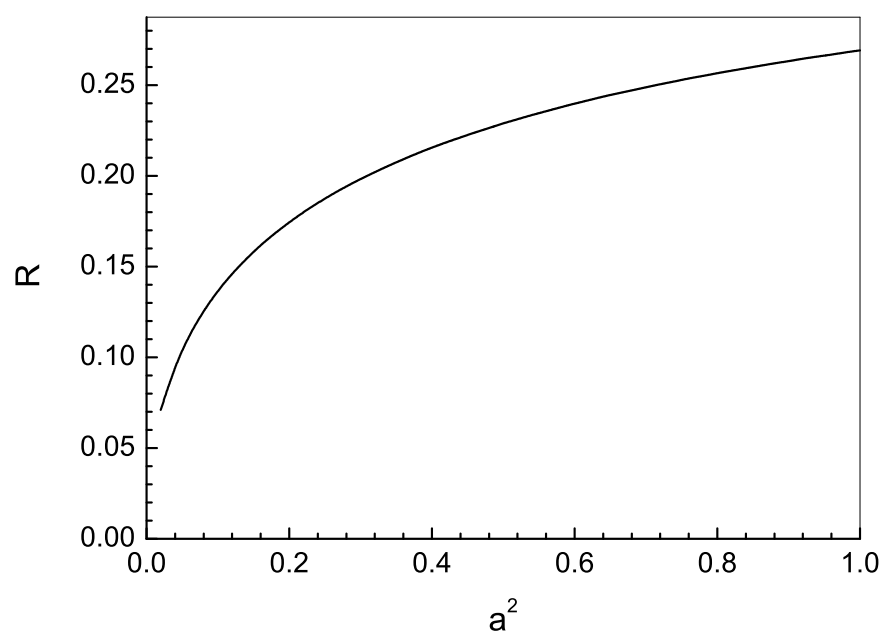

Figure 5: Dependence of $R$ on $a^{2}$ according to Eq.(25) .
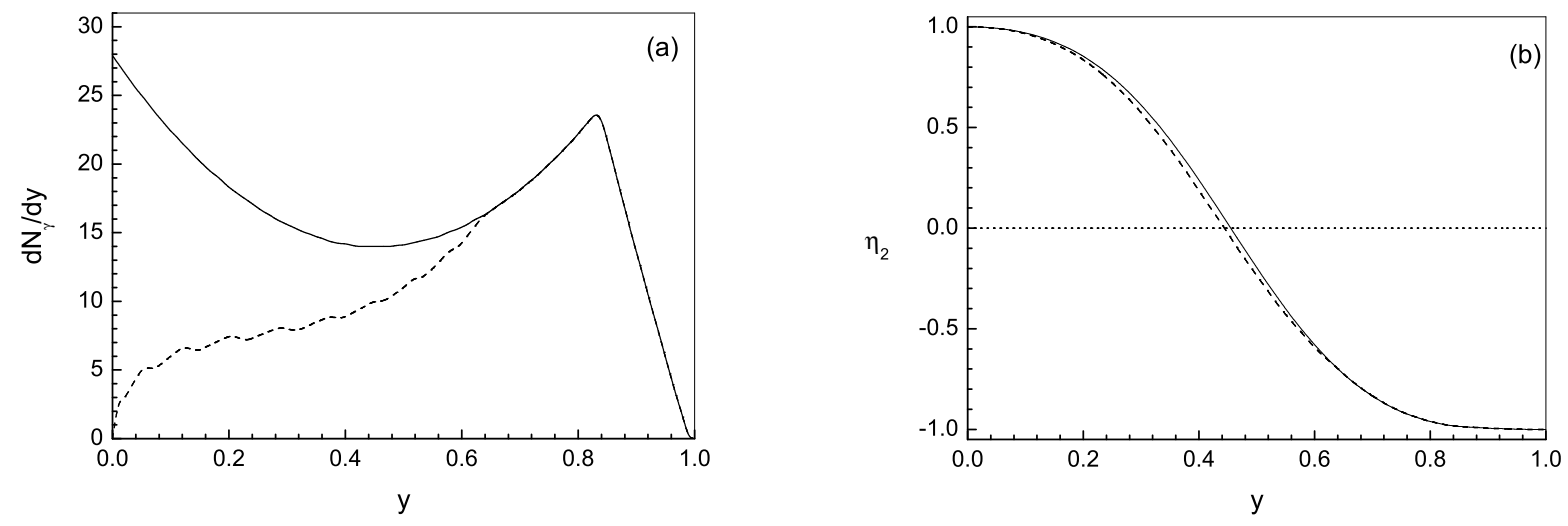

Figure 6: Spectrum $d N_{\gamma} / d y$ and polarization $\eta_{2}^{(\omega)}$ for all generated photons (solid) and for photons which reach the target (dashed); $y=\omega / \varepsilon_{0} / s_{0}, \varepsilon_{0}=5.8 \mathrm{GeV}, s_{0} \equiv s\left(\varepsilon_{0}\right) \simeq 0.01$.

electron) and circular polarization for all generated photons (solid) and for photons which reach the target (dashed) for conditions of [9]. The calculation is performed using Eq.(25)) and taking into account diminishing of the electron energy and the change of the collimation angle, while a slow increase of $\sigma_{e}$ over a collision section and the laser-power loss caused by mirrors are neglected. Comparison of our results with Fig.14(b) in [9] shows that even in its simplest form our approach provides rather accurate estimations. For example, our calculation overestimate the total number of all generated photons by $\sim 2.4 \%$, and that for photons hitting the target by $\sim 2.1 \%$.

\section{Conclusion}

Among various methods of obtaining longitudinally polarized positrons for future linear colliders, the most promising schemes are those using circularly polarized, high-energy photons for positron production. Most effectively such photons are emitted from electrons passing 
through a helical undulator or colliding with the circularly polarized laser wave. Though the physics of the photon-emission process from electrons is the same in both cases, a drastic difference in the periods of motion leads to the corresponding difference in electron energy needed to produce positrons of several tens of $\mathrm{MeV}$, which can be effectively captured and accelerated. While an electron energy of several $\mathrm{GeV}$ is appropriate in the laser case, hundreds of $\mathrm{GeV}$ are needed for undulators. Another advantage of the laser scheme is in easy switching of the photon (and thereby positron) helicity. This option is very useful in experiments. It seems, however, that there are less questions in the construction of undulators, while producing of laser systems with record parameters (very high power, high repetition rate) and rather sophisticated optics is still a challenge. We propose a new, rather simple presentation of known formulas describing the radiation properties in both cases. Our consideration takes into account such factors as a magnitude of the wave intensity, angular divergence in the electron beam, collimation of radiation, and the lateral and temporal profiles of a laser

bunch. The developed description allows one to choose easily a set of parameters optimizing the photon yield.

\section{Acknowledgements}

One of us (V.S.) is thankful for kind hospitality during his stay at IPN - Lyon where a part of this work has been done. He is also grateful to the Russian Fund of Basic Research for partial support of this work by the Grant 03-02-16510.

\section{References}

[1] G. Moortgat-Pick and H.Steiner, EPJdirect C6 (2001)1

[2] R.Alley et al., The Stanford linear accelerator polarized electron source, SLAC-PUB-956489, (1995).

[3] H.Olsen and L.C.Maximon, Phys. Rev. 114 (1959) 887.

[4] A.P.Potylitsin, Nucl.Instrum.Methods Phys. Res. A 103 (1997) 395.

[5] V.N.Baier, R.Chehab and V.M.Katkov, Nucl.Instrum.Methods Phys. Res. A 338 (1994) 156.

[6] V.E.Balakin, A.A.Mikhailichenko, The conversion system for obtaining high polarized electrons and positrons, INP 79-85, Novosibirsk, (1979).

[7] K. Flöttmann, Investigations toward the development of polarized and unpolarized high intensity positron sources for linear colliders, DESY 93-161a, (1993).

[8] A.W. Weidemann, Polarized positrons at a future linear collider and the final focus test beam, SLAC-PUB-10581, (2004).

[9] T.Omori, T.Aoki, K.Dobashi et al., Nucl.Instrum.Methods Phys. Res. A 500 (2003) 232.

[10] M.Goldhaber, L.Grodzins, A.Sunyar. Phys.Rev.106 (1957) 826.

[11] V.N. Baier, V.M. Katkov, and V.M. Strakhovenko, Electromagnetic Processes at High Energies in Oriented Single Crystals, World Scientific Publishing Co, Singapore, 1998. 
[12] V.M. Katkov and V.M. Strakhovenko, JETP 92 (2001) 561.

[13] V.N.Baier, V.M.Katkov and V.M.Strakhovenko, JETP B 53 (1981) 688.

[14] V.M. Strakhovenko, Phys. Rev. A 68 (2003) 042901

[15] I.S. Gradshteyn and I.M. Ryzhik, Table of Integrals, Series, and Products, 4th Edn. Academic Press, New York, (1965) 